\title{
FRESNEL TRANSFORM SPECTROSCOPY
}

\author{
D. G. FALCONER and J. T. WINTHROP \\ Institute of Science and Technology, The University of Michigan, \\ Ann Arbor, Michigan
}

Received 4 January 1965

This letter describes a new method of spectroscopy which is similar to Fourier transform spectroscopy [1] in that a Michelson interferometer is employed as a dispersion element, and differs in that 1 ) the recording of the spectrum involves a Fresnel rather than a Fourier integral and that 2) the reconstruction of the spectrum is carried out by means of a diffraction process involving no focussing elements. The chief advantage in Fresnel transform spectroscopy is that it provides an efficient and rapid means for obtaining the spectrum analysed by a Michelson interferometer.

Spectrometers which employ a Michelson interferometer as a dispersion element have been developed by Connes [2] and others to a degree where they are competing favorably with the traditional methods of spectroscopy. However, one of the most significant problems facing the Fourier transform spectroscopist is the determination of the spectrum from the interferogram. Present optical techniques, although adequate for low resolution work, are not able to accurately effect the inversion operation when both high spectral resolution $\Delta k$ and broad spectral ranges $\left(k_{\max }-k_{\min }\right)$ are involved. On the other hand, modern computer systems (IBM 7090) have sufficient capacity for the operation, but require excessive amounts of computer time (one-hour), even when sophisticated methods of calculation are used [2]. Fresnel transforms spectroscopy evolved from an effort to obtain the simplicity and speed of the optical methods without sacrificing the accuracy of the computer technique.

Fresnel transform spectroscopy is based on the properties of the "Fresnel transform"

$$
\bar{\rho}(x)=\int \rho(k) \exp \left(\mathbf{i} k x^{2}\right) d k
$$

where $\rho(k)$ is the spectral density of the source at the wavenumber $k$. In this method of spectroscopy the interferometric record is obtained by subjecting one arm of the interferometer to a constant acceleration, while moving the re-

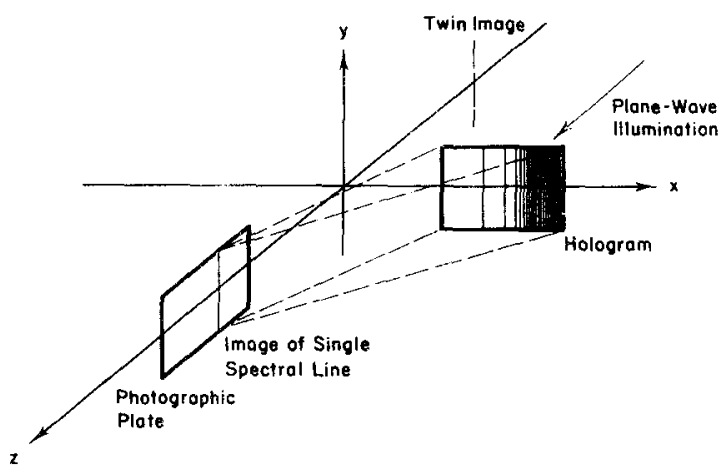

Fig, 1. Lensless reconstruction of the spectrum in Fresnel transform spectroscopy.

cording media at some constant speed. This then produces a recording

$$
H(x)=\bar{\rho}(0)+\bar{\rho}(x)
$$

Thus, if $\rho(k)$ describes a single spectral line, the record is of the form

$$
H(x)=2+\exp +\left(\mathrm{i} k_{0} x^{2}\right)+\exp -\left(\mathrm{i} k_{0} x^{2}\right) .
$$

This, of course, is a one-dimensional cosinusoidal Fresnel zone-plate (focal length $\pi / \lambda k_{0}$ ) centered at $x=0$. The similarity of this record and the ones obtained in wavefront reconstruction [3] is evident; thus, $H(x)$ has been called a hologram.

The spectral density $\rho(k)$ can be obtained from the hologram by means of the optical arrangement shown in fig. 1. The incident plane wave is diffracted by the hologram into three distinct components. In the case of a single spectral line these three components are generated by the three terms of eq. (3); the first term produces an unperturbed plane wave, the second a diverging cylindrical wave, and the third a converging cylindrical wave. Hence, each spectral line is seen to produce an image on the $z$-axis at a distance $\pi / \lambda k_{0}$ from the $x y$-plane. 
The authors wish to acknowledge fruitful conversation with J. Connes regarding Fourier transform spectroscopy, and with G. Stroke regarding wavefront reconstruction.
1. W. Gebbie and G.Vanasse, Nature 178 (1956) 432 .

2. P. Connes, Fourier transform spectroscopy, Lecture delivered at the University of Michigan, Oct. 23, 1964.

3. D. Gabor, Proc.Roy. Soc. (London) A197 (1949) 454.

ON THE HIGH TEMPERATURE SUSCEPTIBILITY

OF THE HEISENBERG MODEL

D. W. WOOD

Department of Mathematics, University of Nottingham, Nottingham, England

Received 4 January 1965

In a recent letter Domb and Wood [1] have developed a new method of deriving high temperature $\left(T>T_{\mathrm{c}}\right)$ power series expansions for the thermodynamic properties of the Heisenberg ferromagnet and antiferromagnet [2]. For the Heisenberg model when the spin value $s=\frac{1}{2}$ the high temperature series expansion for the susceptibility in zero field can be written in the from [3]

$$
\frac{k T}{m^{2}} \mathrm{x}_{0}=1+\sum_{r=1}^{\infty} j_{r} K^{r / 2} 2_{r} \text { ! }
$$

Special methods have been developed $[1,4]$ and high speed digital computers employed to extend previous calculations of the coefficients $j_{\gamma}$. In a previous communication Domb and Wood [1] were able to add two extra terms to the expansion (1) $j_{7}$ and $j_{8}$ ) for the loose packed lattices (diamond, s.c. and b.c.c.). The following term $j g$ has now been derived. It was found that 119 graphs had non zero contributions to this coefficient on the loose packed lattices. It is the purpose of this present letter to present the extra term $j_{9}$ as follows:

$\frac{1}{2} j_{9}=(\sigma+1)\left\{181440 \sigma^{8}-1415520 \sigma^{7}+5201280 \sigma^{6}-10628352 \sigma^{5}+11611584 \sigma^{4}+1190016 \sigma^{3}+\right.$

$\left.-6188736 \sigma^{2}-3957120 \sigma-741952\right\}-p_{4}\left\{14515200 \sigma^{5}-97929216 \sigma^{4}+265798656 \sigma^{3}-246481920 \sigma^{2}+\right.$

$+17326080 \sigma+377856\}+p_{6 a}\left\{9870336 \sigma^{3}-51687936 \sigma^{2}+163123200 \sigma-156492288\right\}-p_{6}\left\{14515200 \sigma^{3}+\right.$

$\left.-78188544 \sigma^{2}+78935040 \sigma+9999360\right\}+p_{7 a}\left\{7451136 \sigma^{2}-6967296 \sigma-21132288\right\}-p_{8}\{9676800 \sigma+$

$-13934592\}+p_{8 c}\{4870656 \sigma-6732288\}+p_{8 r}\{81893376 \sigma-121540608\}+p_{8 h}\{18385920 \sigma-29546496\}+$

$-p_{8 t}\{875520 \sigma-9962496\}+4032000 p_{9 j}+2451456 p_{9 k}+2419200 p_{9 l}+2824704 p_{9 m}-165888 p_{9 s}$.

Here $\sigma=q-1$, where $q$ is the coordination number of the lattice, and $p_{n x}$ are the lattice constants of multiply connected graphs which are tabulated elsewhere [5]. The only lattice constant which is not available elswhere is $p 9 s$ which has the values 0,8 and 56 for the diamond, s.c. and b.c.c. lattices respectively.

I would like to express my thanks to Professor Domb for many helpful suggestions regarding this work, and to Dr.M. F. Sykes for his advice on the configurational aspects of the work. I would also like to thank the London University Computer Unit for their help with computational facilities.

\section{References}

1. C.Domb and D.W. Wood, Physics Letters 8 (1964) 20;

C.Domb and D.W. Wood, in preparation.

2. C.Domb, G.S. Joyce, N.W. Dalton and D.W. Wood, International conference on Magnetism, Nottingham, England, Sept. 1964, to be published. 